\title{
Measuring the Daylight Performance of Classrooms: Can a One Point Sensor Measurement Predict the Daylight Distribution within a Space?
}

\section{Aniebietabasi Ackley}

Victoria University of Wellington, Wellington, New Zealand

aniebietabasi.ackley@vuw.ac.nz

\author{
Michael Donn \\ Victoria University of Wellington, Wellington, New Zealand \\ michael.donn@vuw.ac.nz
}

\section{Geoff Thomas}

Victoria University of Wellington, Wellington, New Zealand

geoff.thomas@vuw.ac.nz

\begin{abstract}
The New Zealand Ministry of Education (MoE) has begun measuring the light, temperature, noise and CO2 level of 21 selected schools using a single sensor. This sensor is being developed as a method for routine measurement in order to understand the performance of New Zealand's school buildings. This study used a Climate Based Daylight Modelling to appraise the MoE methodology, to determine what can be learned from the use of a single sensor in one location in a classroom, to estimate the lighting comfort across a space. Daylighting is focused upon because it has the most spatial variation in a space. The findings of this study support the assertion that a one-point sensor measurement on a vertical wall could predict illuminance across the centre of the horizontal work plane; and provide a useful benchmark to estimate the light distribution across a space. However, regardless of how representative of a space a one-point measurement is, it is difficult to quantify the daylight distribution over time throughout the space. If various daylight indicators are well documented and analysed alongside the measured data, a strategically positioned one-point sensor on the vertical wall could be useful in predicting the daylight quality of a space.
\end{abstract}

Keywords: Daylight, Temperature, Measuring, Sensors, Classrooms

\section{INTRODUCTION}

Daylight is a vital environmental variable that largely define the quality of the indoor environment. Classroom studies (Barrett, Davies, Zhang, \& Barrett, 2015; Heschong Mahone Group, 1999) focusing on skylighting as a way to isolate daylight as an illumination source, and separate illumination effects from other qualities associated with daylighting from windows. It establishes a statistical connection between daylighting and student performance and between skylighting and retail sales. Using multivariate linear regression analysis, the study examined 21,000 school records from 3 school districts in 3 states and daylighting conditions in over 2,000 classrooms. Data indicate students with the most classroom daylighting progressed 20 percent faster on math tests and 26 percent on reading tests in one year than those with the least. Similarly, students with the largest windows progressed 15 percent faster in math and 23 percent faster in reading than those with the least. In classrooms where windows could be opened, there was a 7 to 18 percent faster educational progress than those with fixed windows, regardless of air conditioning. These findings are reported to be consistent regardless of curricula or teaching styles. Appendices provide data charts, a report from one of the classroom-level analyses, sample illumination readings, classroom plans and sections, and photographs of schools and classrooms. (GR have shown that poor daylight could significantly impact on students learning performance and health. The Architectural Energy Corporation (2006) state that, psychological and energy efficiency are the two vital benefits of daylight. The psychological benefit is evident in studies by Heschong Mahone Group, (1999) and Nicklas \& Bailey, (1997), which showed that there was an increase in student test scores in daylight classrooms compared to non-daylight classrooms. A review of indoor environmental quality in schools by; Ackley, Donn, \& Thomas, (2017) suggest that daylight is of primary importance in schools, because it helps to enhance visual comfort, and reduces a building's energy consumption through minimizing the use of electric lighting.

The distribution of daylight within a space differs widely due to the sun movement, differences in seasons and changing weather conditions. Therefore, the proper location of a sensor and the number of sensors required within a space is very important in determining the quality of data derived. In a real-world scenario, there could be many constraints in placing multiple sensors in many classrooms. Hence, measuring illuminance at locations that are more reliable, such as walls, and ceilings and the use of limited number of sensors becomes necessary to use as a proxy for illuminance performance on the work plane. 
This study reports the results of simulating the spatial distribution of the yearly daylight quality of a typical classroom model. The reason for focussing on daylight is because, the depth and building configuration of a space could impact on the distribution of daylight across a space. Also, simulation tools help to calculate the annual daylight performance of a space to predict the illuminance distribution within a space. The goal was to investigate whether a limited number of sensors can reveal the distribution of visual comfort across a space and where might be the most representative placement of a single sensor within a classroom.

\section{LITERATURE REVIEW}

\section{Daylight Sensors in Classrooms}

Light, temperature, noise and $\mathrm{CO} 2$ sensors have been deployed in many school buildings around the world to assess the quality of the indoor environment provided by the building. But the proper location of a sensor within a space such as a classroom could be a difficult task, because the activities of students in the work plane may affect the sensors. This assertion is supported by Mardaljevic, (2001) who state that, "for any occupied space, not a least a school classroom, it is practically impossible to obtain a reliable time-series of illuminance measurements taken at desk height without cordoning off the area around the sensor - an intervention that would be difficult to both approve and enforce". Therefore, it is necessary to consider other means of predicting illuminance on the working plane. Studies (Mardaljevic et al., 2016; Mistrick et al., 2000; Ranasinghe \& Mistrick, 2003) have suggested that aside from the work plane, the walls and ceiling of a classroom could be appropriate to place daylight sensors, these studies have also stated the advantages of properly locating sensors.

Ranasinghe \& Mistrick, (2003) argue that specific illuminance at a task is influenced by the location of a sensor relative to the daylight aperture. They hypothesize that the best performance of a sensor is derived when it is mounted to minimize its view of the window. Also, mounting a sensor with wide spatial sensitivity on the ceiling will make it difficult to track daylight when the sensors receive more direct light from the daylight aperture (Ranasinghe \& Mistrick, 2003). A study by Mistrick \& Thongtipaya, (1997) in a small office setting showed that when a sensor's view of the window is minimized or completely eliminated, there is a higher correlation between the sensor's sensitivity and the daylight level; because, the sensor will receive light from the wall, and the horizontal surface of the space.

On the location of sensors on vertical walls to predict horizontal illuminance, an earlier study by Mardaljevic, (2001), suggest that, measured Illuminance at one or more points on a vertical wall could predict the horizontal illuminance in the working plane provided that the evaluation excludes very high illuminance values. This implies that any instances of direct sun on the work plane or on wall surfaces should be avoided. This assertion is supported in a latter study by Mardaljevic et al., (2016) were they showed that illuminance levels on the wall could be a proxy for work plane illuminance in classrooms. Using the Radiance 2-phase CBDM, they placed sensors in the centre of the work plane of a $11.2 \mathrm{~m}$ by $7.9 \mathrm{~m}$ and $3.0 \mathrm{~m}$ floor to ceiling side-lit classroom, while 10 vertical sensors were placed on the long wall and six on the short wall. They found a 0.97 correlation between the work plane illuminance with that of the wall and the North elevation showed the best correlation. They concluded that, "vertical wall illuminance can serve as a proxy for horizontal desk illuminance", and that the relationship between the illuminance performance of the work plane with that of the wall is distinct to the type of space and can be determined using the Climate Based Daylight Modelling (CBDM). Despite these findings, the validation of predicted measures of daylight performance in classrooms have proven to be a challenging prospect.

To interpret the illuminance values of this study, Useful Daylight Illuminance (UDI) and Daylight Autonomy (DA) are the illuminance-based metrics explored. UDI is defined as the fraction of time in a year when the indoor horizontal daylight illuminance at a given point falls within a given threshold (Mardaljevic and Nabil, 2005). In studies related to schools, the lower UDI threshold is set to 300 lux, which is in line coherently with international recommended standards (Costanzo, Evola, \& Marletta, 2017). In this study, the UDI threshold is set to $>300-<2000$ lux. DA is the percentage of the annual daytime hours of occupancy at a point which is sufficient to exceed a benchmark illumination level (Standard IES LM-83-1., 2012). DA can show that for a significant percentage of the annual working hours, there is adequate daylight in a particular point in a space, but it cannot show if this point underperforms during the winter period or in the morning hours (Kleindienst, Bodart, \& Andersen, 2008). In this study, the DA is set to $>300$ lux. To understand the distribution of daylight in a space over time, combining UDI and DA would produce highly detailed analysis and helps to easily absorb and interpret a large data set of illuminances (Kleindienst, Bodart, \& Andersen, 2008).

\section{METHODOLOGY}

The New Zealand Ministry of Education (MoE) has begun measuring the indoor environmental quality (IEQ - light, temperature, noise and $\mathrm{CO}_{2}$ ) of 21 selected schools using a single sensor. This is the beginning of a plan to develop this type of sensor as a routine measurement in many more schools aimed at collating evidence-based performance data of New Zealand's school buildings to guide investment decisions. A central data logger which simultaneously measures the IEQ variables is placed in one sampling point at the centre of the short wall of a two-side lit classroom and on the long wall of a one-side lit classroom. The measurement protocol also includes monitoring of the daily outdoor temperature. The dataloggers are 
calibrated by environmental experts and researchers to a known 'standard chart tolerance level'.
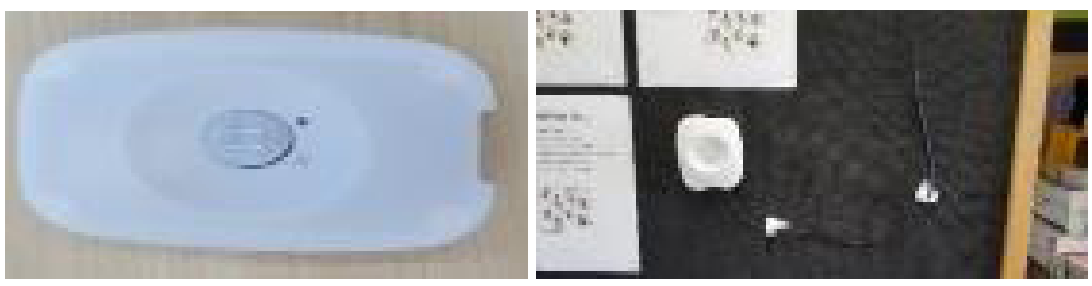

Figure 1: Picture showing one of the MOE data sensor placed in a classroom, Source: (MoE, 2017)

The measurement interval is about 10 times in every $1 \mathrm{~min}$. The data loggers are positioned in accordance with international recommendations (ISO, 2001), avoiding windows and HVAC systems proximity if any. The lighting measurement considered both artificial lighting and daylight together, as it is the actual condition of occupied classrooms during the day. To determine the quality of the artificial lights in each classroom, one-time lighting measurements were taken at night with all windows closed. A central server transmits the measured data wirelessly through a cellular network to an online IEQ database. A 3D camera is used for the visualisation of the interior space of the classrooms, and a drone is used to video the external surroundings and capture the building footprint. Other building daylight indicators are also well documented. Considering that the volume of the MoE environmental data will be enormous, the use of a single measuring point makes it a subject of concern to researchers, scholars, and policy makers. This study uses daylight computer simulation modelling tools to appraise the MoE methodology.

In recent years, computer simulations have proven to provide realistic and reliable data for real world analysis. Studies (Au \& Donn, 2010; Bian \& Ma, 2017; J Mardaljevic, 2000; Reinhart \& Walkenhorst, 2001) support this assertion by stating that simulation provides realistic measures of predicting daylight within a space and can be used for a "real world analysis". The simulation tools used in this study are Grasshopper for Rhino and Honeybee. Grasshopper is a free, parametric modelling graphical algorithm editor, while Honeybee is a free, open source environmental plugin for Grasshopper 3D (Roudsari \& Pak, 2013). The steps used in Grasshopper to run the analysis were; parametrically iterating the geometry for simulation, examining the input file and running the simulations. A Climate Based Daylight Modelling (CBDM) was used to run the simulations of this study, because measuring one moment of daylight may not represent the overall quality of daylight in a space, due to its spatial distribution. CBDM uses realistic sun and sky conditions derived from standardised climate data to predict illuminance (Mardaljevic, 2000; Reinhart \& Herkel, 2000). The climate specific data used for this study was that of Wellington, New Zealand. In Wellington, February is the hottest month with an average temperature of $19^{\circ} \mathrm{C}$ while July is the coldest month with an average temperature of $10^{\circ} \mathrm{C}$. May is the wettest month with an average of $50 \mathrm{~mm}$ of rain (MetService, 2018).

Two classroom iteration design, annotated as Plan A and Plan B were modelled in this study. Plan A (two-side lit) is an empty typical classroom space of length $12 \mathrm{~m}$, width $8 \mathrm{~m}$ with a height of $3.2 \mathrm{~m}$. In New Zealand, typical classrooms are referred to as standardised classrooms, which have a reference design guide and found in most schools across the country. Four continuous sets of windows, each measuring $1.5 \mathrm{~m}$ in width and $1.8 \mathrm{~m}$ high with a sill level of $0.8 \mathrm{~m}$ were located on the $12 \mathrm{~m}$ walls respectively. These windows were on both the North and South-facing walls. Plan B (one-side lit) has the same design configuration of Plan A but the windows were only North Facing. A horizontal calculation grid extended across the work plane of the entire room at a height of $0.8 \mathrm{~m}$ with a $1.6 \mathrm{~m}$ spacing for the columns and rows of the calculation points. On the vertical wall was the one-point measurement sensor located at a $1.5 \mathrm{~m}$ height at the centre of the long and short walls respectively, and replicating the similar position used by the MoE to collect the one-point sensor measured environmental data.

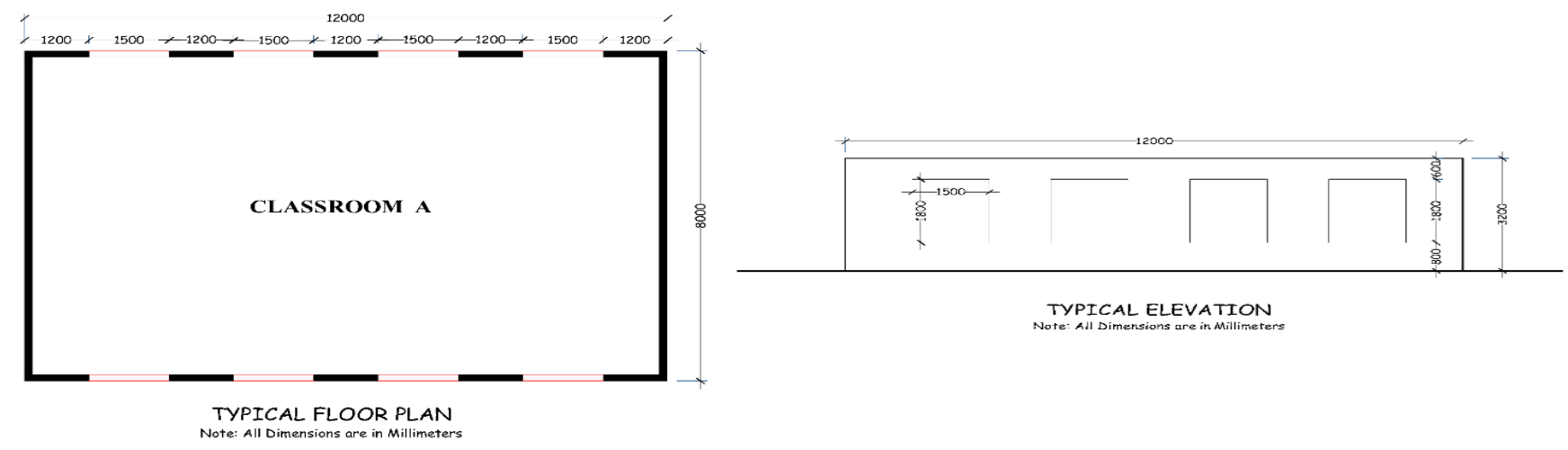

Figure 2: A Typical Plan and Elevation of the Simulated Classroom 
Illuminance values were retrieved at 24 sensor points on the horizontal work plane annotated as A1 to F4 respectively, and from the one-point sensor on the vertical walls. For daylighting distribution evaluation in classrooms, internal illuminance in lux was considered as the most appropriate parameter for indicating the quantity of admitted daylight. It was assumed that both Plan A, and B had no internal and external obstructions such as furniture, partition walls, blinds and occupants, buildings, tree, etc. The ceiling reflectance was 0.7 , wall reflectance 0.5 , and floor reflectance of 0.3 in the space. Light transmittance of all glazing elements was 70\% for adequate daylight transmittance. Neufert, (2000) states that a 1.9 to 2 $\mathrm{m}$ space is required per student in a standard classroom design, hence the classroom should occupy about 40 students.

\section{RESULTS}

Having completed the Climate Based Daylight Modelling, Honeybee stores the annual simulation results in csv-files, the file is opened using Notepad and the data transferred to Excel for evaluation. The researcher determines how the results are displayed visually or numerically. In this study, Sparkline's were used to show illuminance distribution trends in the two iteration plans ( $A$ and $B$ ) respectively. A Sparkline is a tiny chart in the cell of a worksheet that is used to show trends in a series of values by providing a visual representation of the data. Sparklines can readily show patterns in a large set of data to show the relationship between two or more values, comparison of maximum and minimum values, and where there is a decrease or increase in a set of values. In this study, summer time, winter time, annual illuminance of sensor points, and average annual illuminance in the horizontal plane were compared with that of a one-point sensor on the long and short walls (vertical) respectively. Useful Daylight Illuminance and Daylight Autonomy of sensor points on the horizontal plane were also compared with that of a one-point sensor on the long and short walls respectively. The findings of the simulations are reported below.

\subsection{Daylight Distribution In Plan A}

\section{Summer Time Illuminance Distribution}

Figure 3 shows summer time illuminance distribution in sensor points across the horizontal plane of classroom A with that of a one-point sensor measurement on the long and short walls respectively. From the 24 sensor points on the horizontal plane, 20 sensor points (A, B, C, D 1-4, and E1, E2, E4, and F3) showed a relationship with the illuminance pattern of the short and long walls respectively. This relationship was relatively strong in 14 sensor points (B3, B4, C, D, 1-4, and E1, E2, E4, and F3). The centre of the horizontal plane ( $C$ and $D, 1-4)$ showed a very strong relationship with the illuminance trend of the long wall. Only 4 of the 24 sensor points in the horizontal plane showed a different trend from that of the vertical sensors.

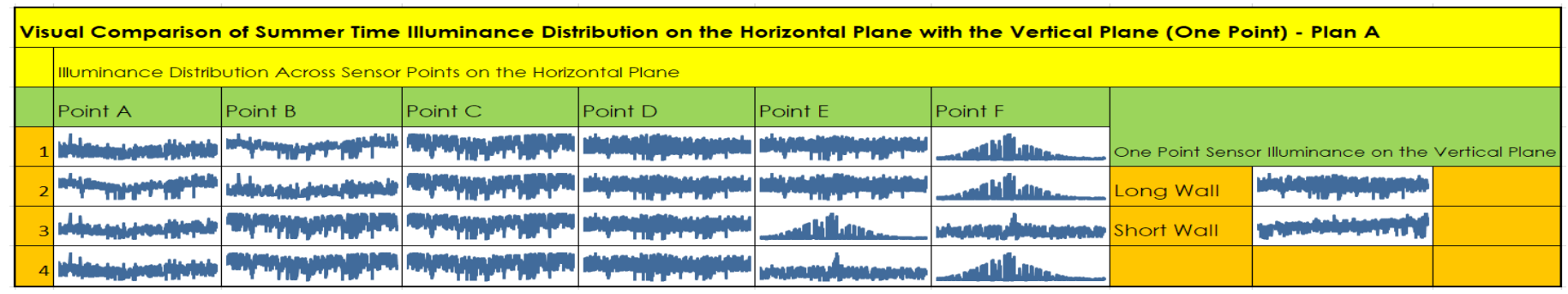

Figure 3: Comparison of Summer Time Illuminance Distribution on the Horizontal Plane with that of a One Point Sensor on the Long and Short Walls respectively 


\section{Winter Time Illuminance Distribution}

In Figure 4, and from the 24 sensor points on the horizontal plane, 13 sensor points (A 1-4, B1, B2, B3, C1, C2, C4 and $\mathrm{F} 1, \mathrm{~F} 2, \mathrm{~F} 4$,) showed a relationship with the illuminance pattern of the short and long walls respectively. This relationship was relatively strong in 4 sensor points (B3, $C 1, C 2$, and $C 4$ ) for the long wall and 6 sensor points (A 1-4 and B 1-2) for the short wall. The centre of the horizontal plane (C1, C2 and C4) showed a very strong relationship with the illuminance trend of the long wall.

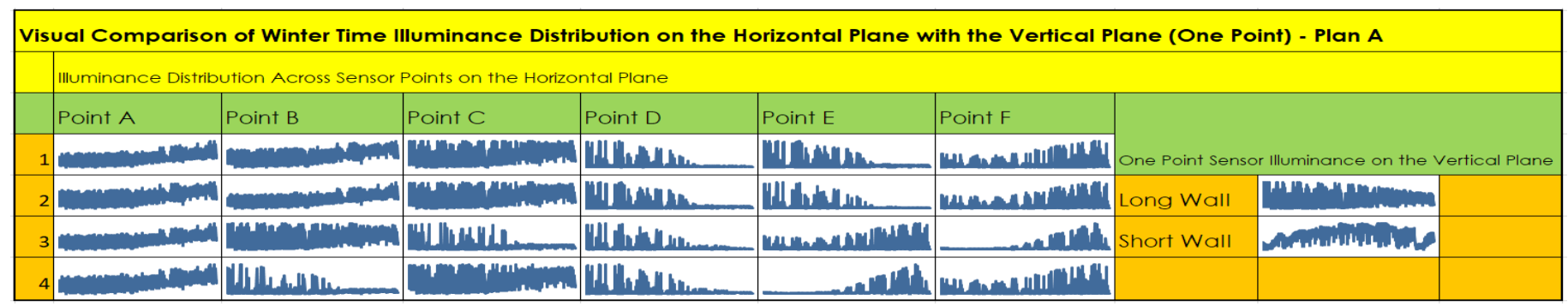

Figure 4: Comparison of Winter Time Illuminance Distribution on the Horizontal Plane with that of a One Point Sensor on the Long and Short Walls respectively

\section{Annual Illuminance Distribution}

In Figure 5, the 24 sensor points in the horizontal plane showed a spatial annual distribution patterns of illuminance. There is a trend between illuminance pattern in 8 sensor points (B3, C1, C2, C4, E3, F1, F2 and F4) on the horizontal plane with that of the long wall, while 6 sensor points (A 1-4, B 1-2) showed a relative trend with that of the short wall. Again, the centre of the horizontal plane (C1, C2 and C4) showed a relatively strong relationship with the illuminance trend of the long wall as well as sensor points F1, F2 and F4 respectively.

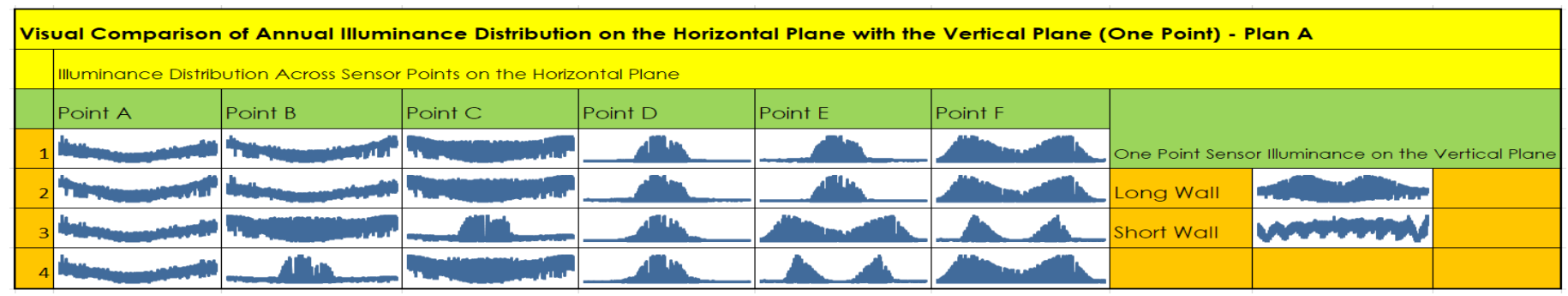

Figure 5: Comparison of Annual Illuminance Distribution on the Horizontal Plane with that of a One Point Sensor on the Long and Short Walls respectively

\subsection{Daylight Distribution In Plan B}

\section{Summer Time Illuminance Distribution}

In Figure 6, and from the 24 sensor points on the horizontal plane, 20 sensor points (A, B, C, D 1-4, and E1, E2, E4, and F3) showed a very strong relationship with the illuminance pattern of the short and long walls respectively. The centre of the horizontal plane (C and D, 1-4) showed the strongest relationship with the illuminance trend of both the long and short wall respectively. Only 4 of the 24 sensor points in the horizontal plane showed a different trend from that of the vertical sensor.

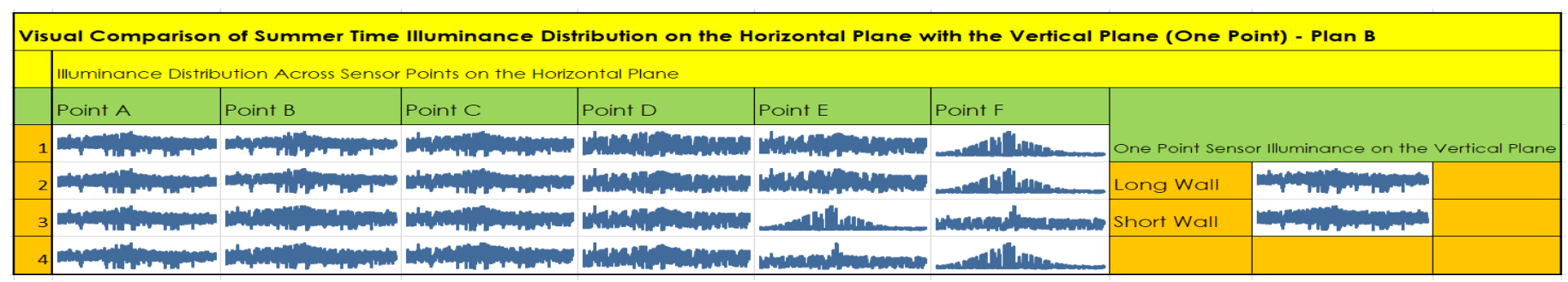

Figure 6: Comparison of Summer Time Illuminance Distribution on the Horizontal Plane with that of a One Point Sensor on the Long and Short Walls respectively 


\section{Winter Time Illuminance Distribution}

In Figure 7, and from the 24 sensor points on the horizontal plane, 14 sensor points $(\mathrm{A} 1-4, \mathrm{~B} 1, \mathrm{~B} 2, \mathrm{~B} 3, \mathrm{C} 1, \mathrm{C} 2, \mathrm{C} 4, \mathrm{E} 3$ and F1, F2, F4,) showed a relationship with the illuminance pattern of the short and long walls respectively. This relationship was very strong in 10 of the 11 sensor points (A 1-4, B1, B2, B3, C1, C2, C3,) for the long wall and the short wall respectively. The centre of the horizontal plane (C, 1, 2 and 4) showed the strongest relationship with the illuminance trend of the vertical walls.

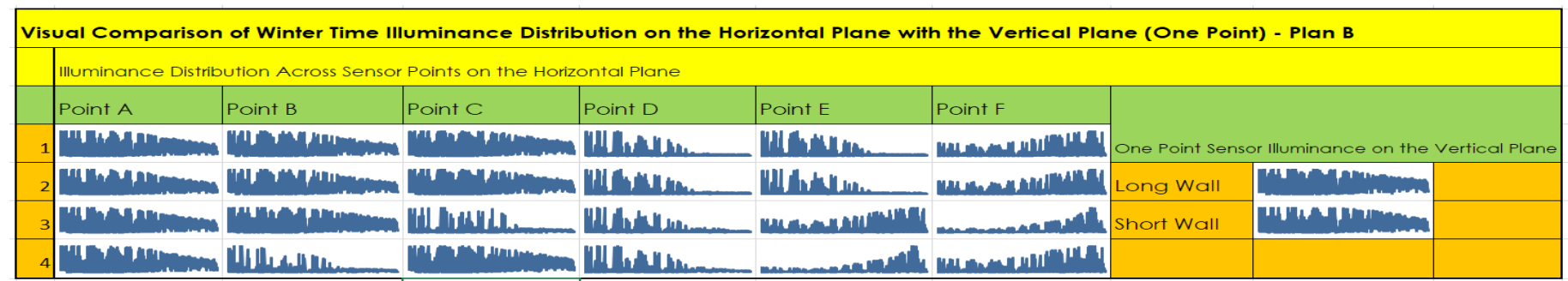

Figure 7: Comparison of Winter Time Illuminance Distribution on the Horizontal Plane with that of a One Point Sensor on the Long and Short Walls respectively

\section{Annual Illuminance Distribution}

In Figure 8, and unlike Plan A that showed spatial annual distribution patterns of illuminance, Plan B showed a trend between illuminance patterns in 14 sensor points (A 1-4, B 1-3, C1, C2, C4, E3, F1, F2 and F4) on the horizontal plane with that of the long wall and short wall respectively. Again, the centre of the horizontal plane $(C, 1,2$ and 4$)$ showed a strong relationship with the illuminance trend of the long and short wall respectively.

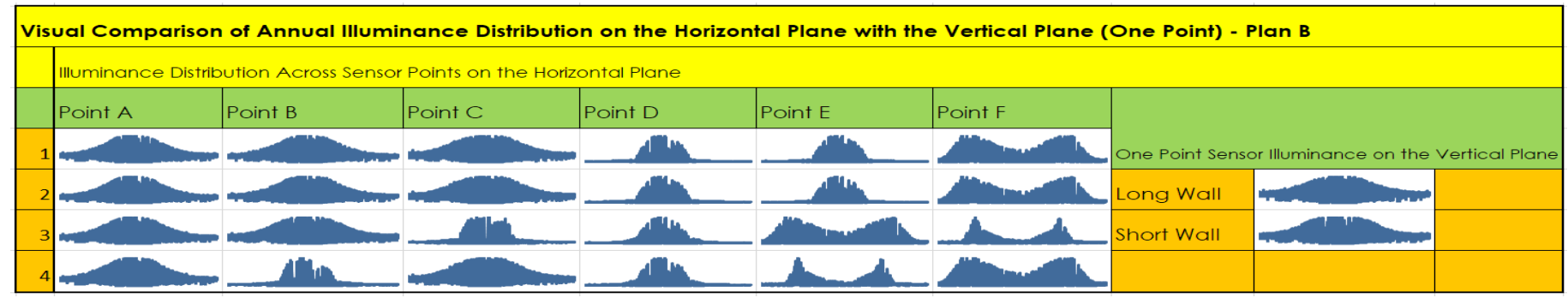

Figure 8: Comparison of Annual Illuminance Distribution on the Horizontal Plane with that of a One Point Sensor on the Long and Short Walls respectively

\subsection{DAYLIGHT AUTONOMY (DA) AND USEFUL DAYLIGHT AUTONOMY (UDI)}

Figure 9 shows a compassion of DA (>300 lux) and UDI (>300<2000 lux) in all 24 sensor points on the horizontal plane with that of the one-point sensor on the long (LW) and short (SW) walls respectively. There was no significant difference in the DA and UDI of sensor points on the horizontal plane with that of the long and short walls respectively. Aside from E3, F1 and F3, all sensor points on the horizontal and vertical walls showed a DA and UDI for more than $50 \%$ of the time. This implies that a large proportion of the classroom had illuminance value within the specified DA and UDI threshold. In classroom B, while some points on the horizontal plane had a DA and UDI trend like that of the vertical walls, other points showed a very different trend. Points A, B and C 1-4 showed DA and UDI for less than 50\% of the time. This implies that a proportion of the classroom had illuminance values that were less than the specified DA and UDI threshold. Generally, and considering that classroom B is a one-side lit classroom, it had lower illuminance values than classroom $A$, which was a two-side lit classroom with higher illuminance values. 


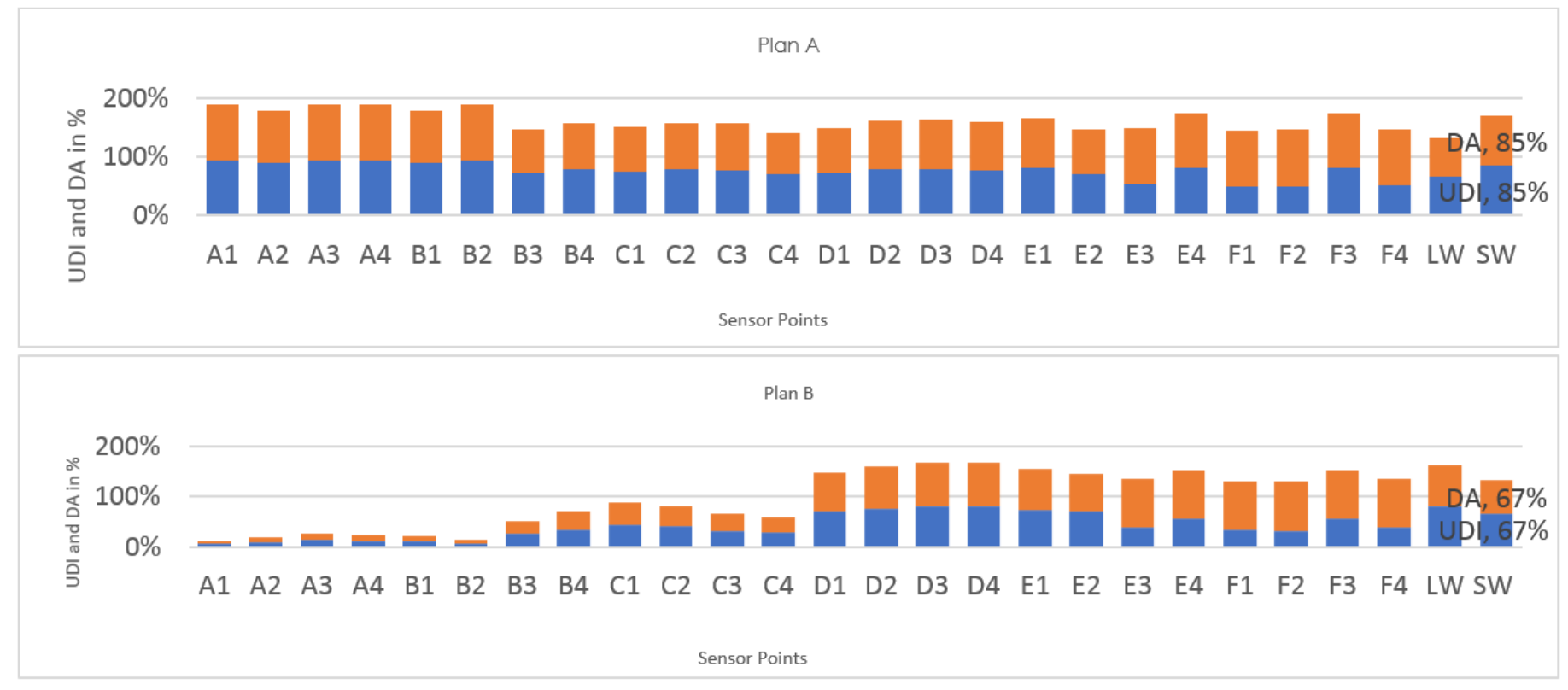

Figure 9: UDI and DA Comparison on the Horizontal Plane and Vertical Walls in Plan A and B respectively

\subsection{ANNUAL AVERAGE ILLUMINANCE DISTRIBUTION}

Figure 10 shows a comparison of annual average illuminance distribution across the 24 sensor points on the horizontal plane with that of the one-point sensor on the long and short wall respectively. In Plan A many points showed a strong relationship between the average illuminance on the horizontal plane and that of the long and short walls respectively. Similarly, plan B also showed the same trend in the average illuminance comparison of the horizontal plane to that of the vertical wall. It is noted that, points E3, F1, F2, and F4 had higher illuminance values than other points in both Plan A and B.

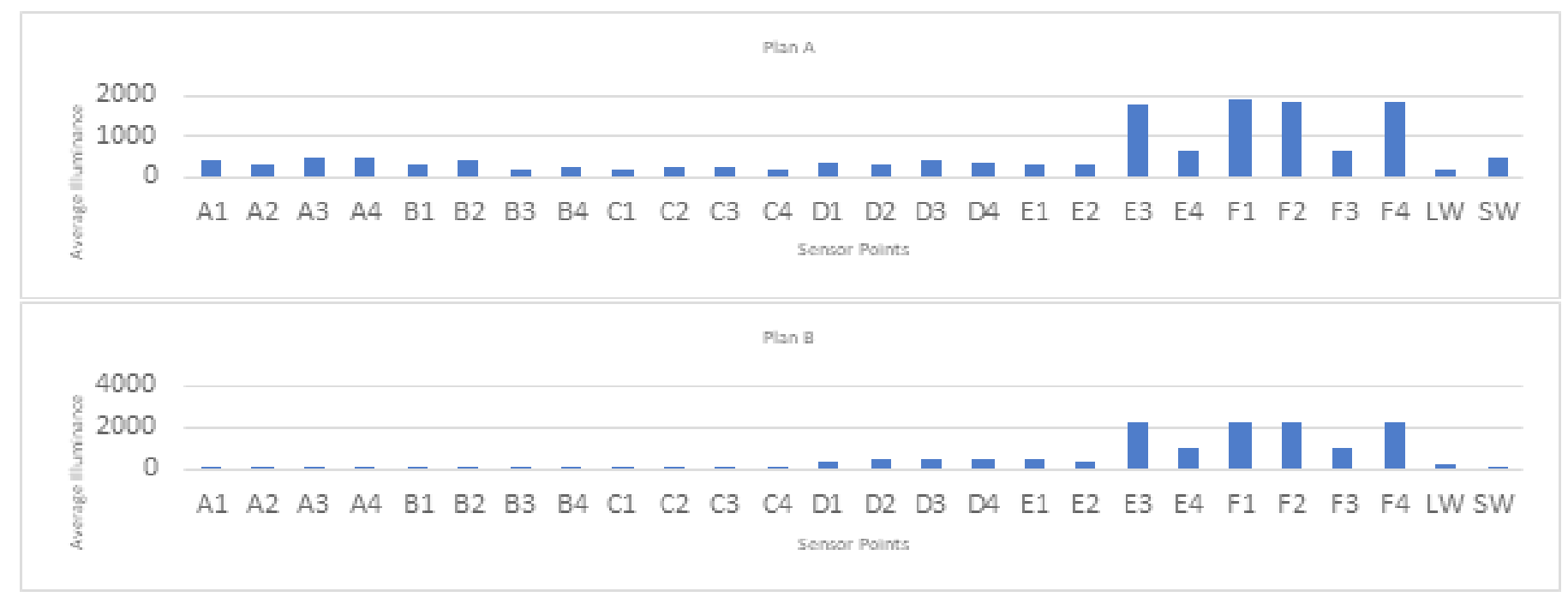

Figure 10: Comparison of Annual Average Illuminance Across Sensor Points in the Horizontal Plane and the Vertical Wall in Plan A and B respectively. 


\subsection{SUMMER TIME ILLUMINANCE COMPARISON}

Figures 3 to 7 showed that illuminance values across the centre (C1) of the horizontal plane had a very strong relationship with that of the long and short walls. Hence, point (C1) at the centre of the horizontal plane of Plan A was plotted with that of the vertical walls to reveal the relationship of these points. Figure 11 showed that there is a strong correlation between illuminance at the centre of the horizontal plane with that of the long wall $(R=0.8)$, and the short wall $(R=0.5)$ respectively. This implies that, illuminance in the vertical plane could predict illuminance at the centre of the horizontal plane.

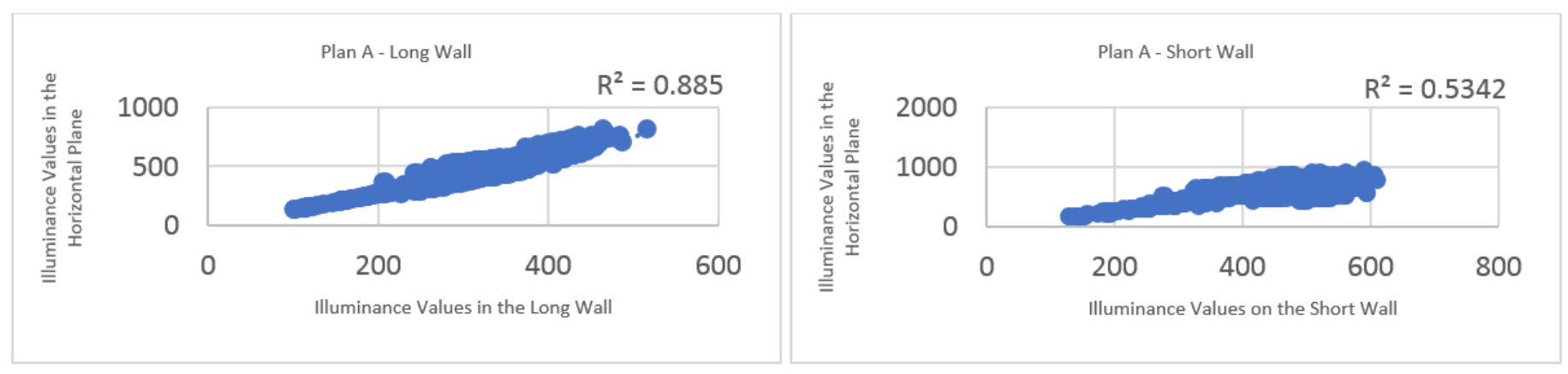

Figure 11: Comparison of Summer Time Illuminance at the Centre of the Horizontal Plane with that of the Vertical Walls.

\section{DISCUSSION}

The results show that illuminance distribution across most points in the horizontal plane, in plan A and B, during the summer and winter periods had a strong relationship with the illuminance trend in the long and short walls respectively. This relationship was significantly stronger in Plan B than $A$ in both summer and winter time respectively, because plan $B$ was a one-side lit classroom, which had lower levels of direct sunlight penetrating deep into the spaces. It was also observed that sensor points on the horizontal plane that showed a trend with sensors on the vertical walls in plan A, were similar to the sensor points in Plan B that showed the same trend. Unlike plan A, plan B showed a stronger annual relationship between sensors on the horizontal plane with the sensors on the vertical wall. Generally, the annual illuminance distribution did not show a very strong correlation in many sensors on the horizontal plane with the vertical sensors on the walls; compared to the strong relationship observed when illuminance values of the summer and winter periods were analysed distinctively. The annual comparisons showed the spatial annual distribution patterns of illuminance. This was observed to be due to high illuminance values, mainly near the windows. This reveals that, even small variations in sensor points relative to the daylight aperture could produce significant differences in the illuminance values.

From the analysis of UDI and DA, it was observed that there was no significant difference in the DA and UDI of sensors across the horizontal plane with the one-point sensor on the long and short walls respectively. While sensor points in Plan A showed illuminance values that were within the DA and UDI threshold, sensor points in plan B showed that many illuminance values were far below the specified DA $(>300)$ and UDI $(>300<2000)$ thresholds respectively. The annual average illuminance trend in sensor points on the horizontal plane were significantly correlated with sensor points on the long and short walls respectively. From the results of summer time comparison between illuminance on the horizontal work plane and that of the vertical wall, it can be inferred that there is a correlation between illuminance at the centre of the horizontal plane and the illuminance of the one-point sensor on the long and short walls respectively. The sensors closer to the windows showed a wide variation in the resulting daylight distributions. Due to the significant amount of direct daylight from the window, the sensors closer to the window had a poorer correlation between the illuminance of the single point sensor on the wall and that of the horizontal work plane (points that admitted direct sunlight deviated from the trend line). However, the agreement between the single point sensor on the wall and the work plane illuminance improves in the sensors that are sufficiently distanced away from the window. These findings agree with the literature which stated that, sensors positioned more distanced from the window performed better. These findings reveal that, illuminance values derived from a onepoint sensor mounted at the centre of the vertical wall (long or short) can be very good for predicting the illuminance on the horizontal work plane (direct light from windows is limited), which could be useful in understanding and estimating the distribution of light within a space.

The scenario described by these results are that;

- if a one-point sensor is mounted at the centre of a vertical wall, and depending on the building configuration (a two-side lit classroom, and a one-side lit classroom respectively), the illuminance values derived could predict the illuminance values across the centre of the horizontal work plane; 
- if for example, the illuminance values derived from the one-point sensor measurement on the vertical wall is assumed to be 350 lux, it could be estimated that a similar range of illuminance value will be derived across the centre of the horizontal plane - provided very high illuminance values are filtered;

- using this assumption, it could be estimated that points away from the centre of the horizontal plane and nearer to the windows would have higher levels of illuminance value than the value derived from the one-point sensor depending on the distance to the daylight aperture;

- the points farer away from the window especially in a one-side lit classroom will have lower illuminance values;

- applying this estimation principle with a detailed analysis of the building design characteristics and site-specific conditions, a one-point measurement on the vertical wall could provide a useful benchmark to estimate the quality of daylight within a space.

For the next stage of this investigation, the objective will be to compare physical data derived from placing multiple data loggers in an experimental occupied and unoccupied classroom with that of a one-point sensor measurement on the four walls. Also, the distribution of temperature, noise and $\mathrm{CO}_{2}$ will be investigated. If reasonable association can be made between measured illuminance data on multiple points in the horizontal plane, and a one-point sensor measurement on the vertical walls, then that would support the proposition that a limited number of sensors could predict daylight distribution within a space.

\section{CONCLUSION}

This study investigated the research question of: can a one-point sensor measurement predict the daylight distribution within a space? Using an advanced climate based daylight modelling, the methodology used by the New Zealand Ministry of Education to collate environmental data in classrooms was explored. The results support the assertion that a one-point sensor measurement on a vertical wall could predict the illuminance values across the centre of the horizontal work plane; and provide a useful benchmark to estimate the daylight distribution within a space. This study has revealed a range of critical considerations inherent in a one-point daylight measurement; by exploring different daylighting trends and patterns, to show the possibility of using limited sensors to measure real-world feasibility. However, the variance in the annual illuminance distribution (Figures 6 and 9) suggests that, it is difficult for a single point in a space to be representative of the daylight quality in an entire space, given the varying nature of daylight indicators such as; the position of the sun in a point in time, building orientation, form, reflectance's of the interior surfaces, window configuration, glass transmission, reflection from nearby deciduous vegetation, and seasonal changes in shading. Also, the actual conditions of an occupied classroom such as the use of artificial lighting may differ from some of the assumptions of the simulated typical model. Therefore, it can be concluded that, regardless of how representative of a space a one-point measurement is, it is difficult to quantify how well daylight is distributed overtime in a space. If the various daylight indicators are well documented and analysed alongside the measured one-point data, a strategically positioned one-point sensor on the vertical wall could be useful in predicting the daylight quality of a space.

\section{References}

Ackley, A., Donn, M., \& Thomas, G. (2017). The Influence of Indoor Environmental Quality in Schools A Systematic Literature Review. 51st International Conference of the Architectural Science Association (ANZAScA)) (pp. 625-634). Wellington.

Architectural Energy Corporation. (2006). "Daylighting metric development using daylight autonomy calculations in the sensor placement optimization tool." Architectural Energy Corporation, 1-52.

Au, P., \& Donn, M. (2010). HDR luminance measurement: comparing real and simulated data. 46th Annual Conference of the Architectural Science Association, (March), 1-8.

Barrett, P., Davies, F., Zhang, Y., \& Barrett, L. (2015). The impact of classroom design on pupils' learning: Final results ofaholistic, multi-level analysis. Building and Environment, 89, 118-133.

Costanzo, V., Evola, G., \& Marletta, L. (2017). A Review of Daylighting Strategies in Schools: State of the Art and Expected Future Trends. Buildings, 7(2), 41.

Heschong Mahone Group. (1999). Daylighting in Schools. Submitted to George Loisos The Pacific Gas and Electric Company on behalf of the California Board for Energy Efficiency Third Party Program.

Kleindienst, S., Bodart, M., \& Andersen, M. (2008). Graphical Representation of Climate-Based Daylight Performance to Support Architectural Design,. LEUKOS, 5(1), 39-61.

Mardaljevic, J. (2000). Simulation of annual daylighting profiles for internal illuminance. In CIBSE Daylight Group/Society of Light and Lighting Conference on Daylight Design and Research (pp. 111-118). London.

Mardaljevic, J. (2001). The BRE-IDMP dataset: a new benchmark for the validation of illuminance prediction techniques. Institute of Energy and Sustainable Development, 33, 117-134. 
Mardaljevic, J., Brembilla, E., \& Drosou, N. (2016). Real-World Validation of Climate-Based Daylight Metrics: Mission Impossible? CIBSe Technical Symposium, 1-12.

Mardaljevic, J., \& Nabil, A. (2005). The Useful Daylight Illuminance Paradigm: A Replacement for Daylight Factors. In Lux Europa, Berlin (pp. 169-174). the conference.

MetService. (2018). Wellington Metrological Data. Retrieved from http://www.metservice.com/towns-cities/wellington/wellington-city\#!/ ten-day

Mistrick, R., Chen, C. H., Bierman, A., \& Felts, D. (2000). A comparison of photosensor-controlled electronic dimming systems in a small office. Journal of the Illuminating Engineering Society, 29(1), 66-80.

Mistrick, R., \& Thongtipaya, J. (1997). Analysis of daylight photocell placement and view in a small office. Journal of the Illuminating Engineering Society, 26(2), 150-160.

Neufert, E. (2000). Neufert Architects Data. London: Malden, MA : Blackwell Science Publishers.

Ranasinghe, S., \& Mistrick, R. (2003). A study of photosensor configuration and performance in a daylighted classroom space. Journal of the Illuminating Engineering Society, 32(2), 3-20.

Reinhart, C. F., \& Herkel, S. (2000). The Simulation of annual daylight illuminance distributions-a state-of-the-art comparison of six RADIANCE-based methods. Energy and Buildings, 32(2), 167-187.

Roudsari, M., \& Pak, M. (2013). Ladybug: a Parametric Environmental Plugin for Grasshopper To Help Designers Create an Environmentally-Conscious Design. 13th Conference of International Building Performance Simulation Association, 3129-3135.

Standard IES LM-83-1. (2012). Approved Method: IES SpatialDaylight Autonomy (sDA) andAnnual Sunlight Exposure (ASE). Illuminating Engeneering Society: New York, NY, USA,. 